\title{
Propiedades geoquímicas y magnéticas de sedimentos como indicadores de contaminación. Caso de estudio: río Suquía, Córdoba, Argentina
}

\section{Laura Daniela Sepúlveda ${ }^{1, *}$, Karina Leticia Lecomte ${ }^{1,2}$, Andrea Inés Pasquini ${ }^{1,2}$, Estefania Gisele Mansilla ${ }^{3}$ y Marcos Adrián Eduardo Chaparro ${ }^{3}$}

\author{
${ }^{1}$ Centro de Investigaciones en Ciencias de la Tierra (CICTERRA), Consejo Nacional de Investigaciones \\ Científicas y Técnicas (CONICET) y Universidad Nacional de Córdoba, Av. Vélez Sarsfield 1611, X5016CGA, Córdoba, Argentina. \\ ${ }^{2}$ Facultad de Ciencias Exactas, Físicas y Naturales, Universidad Nacional de Córdoba, \\ Av. Vélez Sarsfield 1611, X5016CGA, Córdoba, Argentina. \\ ${ }^{3}$ Centro de Investigaciones en Física e Ingeniería del Centro de la Provincia de Buenos Aires, \\ Universidad Nacional del Centro de la Provincia de Buenos Aires (CIFICEN, CONICET - UNCPBA), Tandil, Argentina. \\ *sepulveda.dlaura@gmail.com
}

\section{RESUMEN}

La cuenca del río Suquía (superficie $\sim 6300 \mathrm{~km}^{2}$ ) es un sistema hidrológico de mediana extensión, ubicado en la provincia de Córdoba, en la región central de Argentina. El río Suquía nace del lago San Roque, discurre por sectores serranos, atraviesa una ciudad densamente poblada (Córdoba: 1.5 millones de habitantes) y continúa su curso por la llanura Chaco-Pampeana donde se desarrollan actividades agrícolas y ganaderas. Debido a diversas actividades urbanas e industriales, el río recibe, a lo largo de su curso, aportes contaminantes, los cuales se depositan aguas abajo, en el lecho del mismo. Los resultados de los análisis geoquímicos y magnéticos de los sedimentos depositados en el lecho principal del río, permitieron evaluar el grado de contaminación de este sistema natural. Se extrajeron muestras de sedimento de fondo del cauce del río y se separaron distintas fracciones granulométricas para determinaciones magnéticas. Se utilizaron métodos de magnetismo ambiental (susceptibilidad magnética y magnetización remanente anhistérica) para la identificación y cuantificación de minerales magnéticos, entre ellos, ferrimagnéticos del tipo magnetita. En la fracción limo-arcilla $(<62.5 \mu \mathrm{m})$ se determinaron óxidos mayores y elementos traza. Desde el punto de vista geoquímico los sedimentos evidencian un bajo grado de madurez mineralógica y son equivalentes a lutitas y lutitas ricas en Fe. Los indicadores de contaminación (Índice de Geoacumulación, $\mathrm{I}_{\text {geo }}$; Factor de Enriquecimiento, FE; Contaminación Urbana Industrial, C.U.I.) arrojaron los valores más altos en el área serrana, donde también se registraron los mayores valores de susceptibilidad magnética, debido a factores naturales potenciados por la explotación de canteras. En el área urbana se registraron los valores más bajos de contaminación, mientras que, en la zona rural, se determinó un grado de contaminación moderado a alto, asociado a las actividades agrícolas propias del uso de la tierra. De este modo, los procesos que dan origen a las características geoquímicas y magnéticas de estos sedimentos se diferencian espacialmente a lo largo de la cuenca y se deben a factores tanto naturales como antrópicos. Además, las concentracio- nes de metales en los sedimentos se han incrementado en la última década en toda la cuenca. La correlación significativa entre variables geoquímicas y parámetros magnéticos demuestra la potencialidad de estudios combinados como una metodología de monitoreo ambiental en sistemas hídricos.

Palabras clave: sedimentos fluviales; índices de contaminación; metales; magnetismo ambiental; monitoreo ambiental; río Suquía; Córdoba, Argentina.

\section{ABSTRACT}

The Suquía river basin (area $\sim 6300 \mathrm{~km}^{2}$ ) is a medium-sized hydrological system located in the province of Córdoba, in the central region of Argentine. The Suquia river derives from the San Roque lake. This river flows through mountainous areas, crosses a densely populated city (Córdoba: 1.5 million inhabitants) and continues its course through the Chaco-Pampean plain, where agriculture and livestock farming are typical anthropic activities. The Suquía river receives along its course diverse polluting contributions. The degree of contamination of this natural system was evaluated through geochemical and magnetic analyses. For this purpose, sediment samples were collected from the river bed. The environmental magnetism methods (magnetic susceptibility and anhysteretic remanent magnetization) were carried out in order to determine and quantify the magnetic minerals. Major oxides and trace elements were determined in the $<62.5 \mu \mathrm{m}$ fraction. The sediments show a low degree of mineralogical maturity and are equivalent to shales and Fe-shales. Diverse contamination indices showed the highest values in the mountainous area where the most elevated measurements of magnetic susceptibility were also registered. This is interpreted as consequence of natural factors enhanced by quarries exploitation. The lowest levels of pollution were recorded in the urban area, while in the rural area, a moderate to high degree of pollution associated 
with agricultural activities was determined. Thus, the processes that originate the geochemical and magnetic characteristics of these sediments are spatially differentiated throughout the basin and are due to the effect of both natural and anthropogenic factors. In addition, metal concentrations have increased in the last decade throughout the basin. A significant correlation between geochemical variables and magnetic parameters was determined, proving the potential of combined studies as a methodology for environmental monitoring in hydrological systems.

Key words: fluvial sediment; contamination indices; metals; environmental magnetism; environmental monitoring; Suquía river; Córdoba; Argentina.

\section{INTRODUCCIÓN}

Los componentes químicos transportados en forma disuelta y en partículas por los sistemas fluviales, son el resultado de una serie de factores geológicos (litología, geomorfología, suelo), climáticos (precipitación, temperatura) y numerosos procesos físicos, químicos y bioquímicos. Sin embargo, las actividades humanas causan múltiples y complejos cambios en la química de los ríos. Por ejemplo, la concentración de metales y minerales magnéticos en un río es muy sensible a las diversas actividades antrópicas, tales como la minería y la industria, así como el impacto de las aguas residuales urbanas y los fertilizantes utilizados en la agricultura. Debido a que las acciones humanas en las áreas urbanas aumentan los aportes de nutrientes, metales, compuestos orgánicos y otros posibles contaminantes, los ríos urbanos normalmente generan mayores cargas y concentraciones más variables de contaminantes químicos que los de las cuencas hidrográficas no perturbadas (Meybeck, 2011).

Los sedimentos de fondo pueden constituir un importante agente captador de contaminantes a través de diversos procesos físicos, químicos y/o biológicos. Diferentes enfoques han sido utilizados en la literatura para analizar el grado de contaminación a partir de datos geoquímicos de sedimentos. Los estudios de acumulación o enriquecimiento de metales se basan en técnicas geoquímicas estandarizadas que permiten calcular índices numéricos para cuantificar el grado de contaminación de sedimentos. Otro enfoque más reciente, orientado al estudio de la contaminación en sedimentos, polvo atmosférico y suelos, está referido al análisis de sus propiedades magnéticas. Las propiedades de los minerales presentes en sedimentos y su relación con parámetros magnéticos han sido tradicionalmente de interés en una amplia variedad de estudios, como la interpretación de procesos ambientales naturales (Snowball et al., 2002; Liu et al., 2012; Quijano et al., 2014; Lecomte et al., 2016; Chaparro et al., 2017a). Sin embargo, recientemente han empezado a utilizarse las propiedades magnéticas para la evaluación de la contaminación antrópica (Chaparro et al., 2012; Mejía-Echeverry et al., 2018). Ésta suele tener una fuerte señal magnética y se ha demostrado que es posible discriminar entre diferentes fuentes de contaminación (Chaparro et al., 2006). Algunos trabajos recientes referidos al tema son los realizados por Yang et al. (2007), Franke et al. (2009), Chaparro et al. (2015, 2017b), entre otros.

El río Suquía constituye un sistema hidrológico de mediana extensión que abastece de agua potable a gran parte de la ciudad de Córdoba. Este río está sujeto a procesos de contaminación como consecuencia de efluentes urbanos e industriales, así como de actividades agrícolas. Algunos estudios han sido llevados a cabo en el cauce medio del río Suquía, la mayor parte de ellos orientados al estudio de la hidroquímica y la calidad del agua (Pesce y Wunderlin, 2000; Wunderlin et al., 2001; Valentinuzzi et al., 2006; Pasquini et al., 2012, entre otros), o aspectos biológicos de la contaminación. Asimismo, Pasquini et al. (2012) han señalado que los metales pesados y demás contaminantes inorgánicos potenciales se encuentran en la fase disuelta, en concentraciones similares a aquellas propias de las áreas casi prístinas de la cuenca alta. Los antecedentes referidos a la geoquímica de la fase sólida transportada por el río Suquía son menos abundantes en la bibliografía (Gaiero et al., 1997; Merlo et al., 2011; Stupar et al., 2013 y Hargunteguy et al., 2014). Wunderlin (2018) expuso el conocimiento más avanzado en relación con la contaminación en el agua y en los sedimentos del río Suquía y sus efectos sobre la biota.

El objetivo de este trabajo es utilizar diversas herramientas y técnicas geoquímicas y magnéticas para analizar y evaluar el grado de contaminación de los sedimentos de fondo transportados en la cuenca media del río Suquía. Las herramientas utilizadas incluyen la caracterización geoquímica, el cálculo de índices de calidad y el análisis de las propiedades magnéticas, a fin de realizar un aporte complementario que contribuya a la evaluación del estado ambiental del área de estudio.

\section{ÁREA DE ESTUDIO}

La cuenca del río Suquía se ubica en el centro de Argentina, específicamente en el centro-norte de la provincia de Córdoba, entre los paralelos $30^{\circ} 58^{\prime} \mathrm{S}$ y $31^{\circ} 37^{\prime} \mathrm{S}$ y los meridianos $60^{\circ} 23^{\prime} \mathrm{W}$ y $64^{\circ} 51^{\prime} \mathrm{W}$ (Figura 1). Tiene una extensión de $\sim 6300 \mathrm{~km}^{2}$ de los cuales $\sim 45 \%$ corresponde a su cuenca activa en el área serrana, casi prístina, localizada en las Sierras Grandes de Córdoba ( 2000 m s.n.m.). La región montañosa drena sus aguas a un embalse artificial, el lago San Roque. A partir del lago San Roque nace, con ese nombre, el río Suquía sobre el faldeo oriental de las Sierras Chicas. A $\sim 15 \mathrm{~km}$ aguas abajo del embalse, el río atraviesa la ciudad de La Calera, donde se asientan industrias de cal y molienda de roca granítica que son una de las mayores productoras de materiales de construcción de la provincia. A $\sim 10 \mathrm{~km}$ aguas abajo de La Calera, el río Suquía atraviesa la ciudad de Córdoba, donde se desarrollan principalmente actividades de servicio y comercio, y en menor medida industriales (particularmente la automotriz). El auge de la construcción durante los últimos años ha causado una notable expansión del tejido urbano, así como un gran crecimiento de la industria relacionada con la construcción. Aguas abajo del centro urbano de Córdoba $(\sim 50 \mathrm{~km})$ se encuentran numerosas comunas donde la principal actividad económica es la agricultura y la ganadería.

El caudal medio anual del río Suquía es de $9.7 \mathrm{~m}^{3} \mathrm{~s}^{-1}$, con un derrame promedio de $0.3 \mathrm{~km}^{3}$ año ${ }^{-1} \mathrm{y}$ un caudal específico promedio de $7.2 \mathrm{~L} \mathrm{~s}^{-1} \mathrm{~km}^{-2} a \tilde{n} o^{-1}$ (Dasso et al., 2014). Litológicamente, la región de estudio se caracteriza por un complejo metamórfico compuesto por gneis y migmatita, afectado por la intrusión de un granito paleozoico en el área serrana (Gordillo, 1972; Gordillo y Lencinas, 1979), mientras que, en la cuenca media y baja, afloran rocas sedimentarias continentales y sedimentos de tipo loess (Figura 1). Rapela et al. (1998) y Locati et al. (2009) reconocen una franja de cizalla dúctil, nombrada La Estanzuela, ubicada en la zona de Las Bateas, la cual divide la región de estudio en dos zonas con dominios litológicos contrastantes. Hacia el este aflora el grupo La Calera compuesto por diatexita cordierítica, gneis biotíticogranatífero, mármol calci-dolomítico y anfibolita ricas en diópsido. Hacia el oeste de esta falla, se encuentra el grupo El Diquecito integrado por ortogneis piroxénico, ortogneis tonalítico a granodiorítico y en menor medida, rocas metasedimentarias. En cuanto a las rocas sedimentarias, éstas comprenden las formaciones Saldán, Estancia Belgrano, General Paz y depósitos modernos, cuya fracción arenosa está compuesta principalmente por cuarzo, plagioclasa, oligoclasa, 


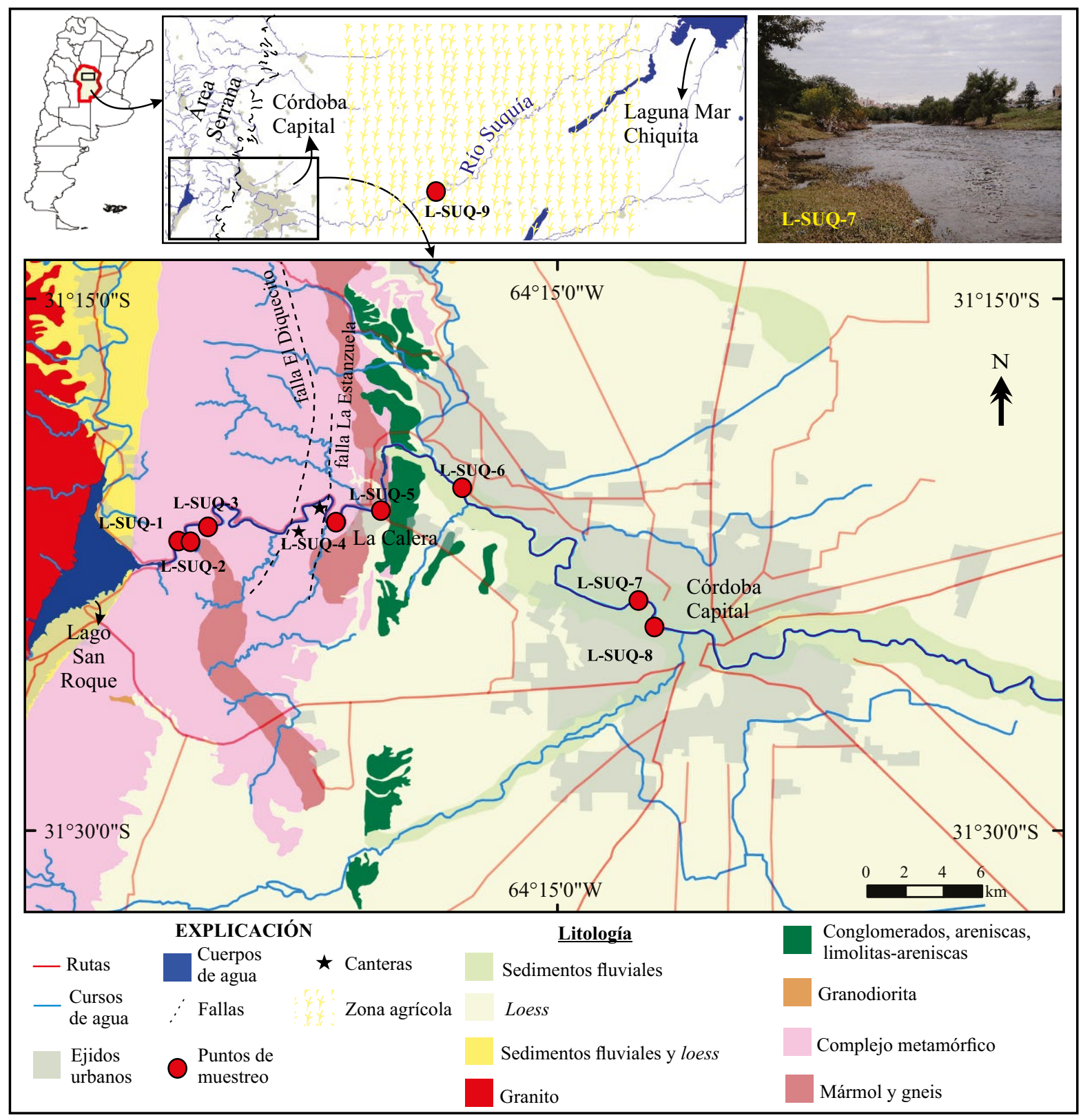

Figura 1. Ubicación de la cuenca del río Suquía, características litológicas y localización geográfica de los puntos de muestreo.

microclina y ortoclasa. Dentro de los minerales pesados predominan el granate y opacos con cantidades subordinadas de epidota, hornblenda, apatita y circón. La formación General Paz está compuesta de loess, además de cantidades regulares de vidrio volcánico (Santa Cruz, 1972).

El clima en la región es templado, semi-húmedo a semiárido, con inviernos secos, tipo $\mathrm{Cw}$ de acuerdo con la clasificación de KöppenGeiger (Kottek et al., 2006). La temperatura media anual oscila entre 14 y $17^{\circ} \mathrm{C}$, y la distribución irregular de las precipitaciones anuales es una característica típica. La precipitación media anual (período 19602015), fue de $815 \mathrm{~mm}$, concentrada en la estación húmeda (el $80 \%$ de la precipitación total ocurre durante el verano austral, entre los meses de noviembre y abril: Servicio Meteorológico Nacional). Debido a su localización geográfica y a las características geológicas y climáticas, la región se caracteriza por un régimen erosivo definido como "limitado por la meteorización" en el sentido de Carson y Kirkby (1972), como fue señalado por Campodonico et al. (2014) y referencias allí incluidas. Los escenarios limitados por la meteorización son aquellos en los que el transporte de materiales es más rápido que la tasa de alteración química. Como resultado, una porción significativa del regolito generado se moviliza rápidamente mediante la acción de los cursos de agua, los vientos y la gravedad.

\section{METODOLOGÍA}

\section{Muestreo y tratamiento de muestras}

Se recolectaron nueve muestras de sedimentos de fondo, durante abril de 2014, a lo largo de la cuenca media del río Suquía, desde el lago San Roque hasta la localidad de Río Primero (Figura 1). Las muestras L-SUQ-1, L-SUQ-2 y L-SUQ-3 se tomaron aguas abajo del lago San 
Roque, en un sector suburbano, donde el río mantiene características de un río serrano, con una significativa pendiente y rocas en su lecho. La muestra L-SUQ-4 fue extraída en la zona de la cizalla dúctil La Estanzuela (campamento Las Bateas), en las proximidades de las canteras de áridos (rocas, arena y grava) que se explotan en la actualidad. Aguas abajo se extrajo la muestra L-SUQ-5, en la región urbanizada de la localidad de La Calera. La muestra L-SUQ-6 fue tomada en Villa Warcalde, al noroeste de la ciudad de Córdoba, mientras que las muestras L-SUQ-7 y L-SUQ-8 fueron extraídas en la zona céntrica y más poblada de la ciudad (puente Tablada y puente Santa Fe, respectivamente). Por último, la muestra L-SUQ-9 se extrajo en la localidad de Río Primero, una región rural caracterizada por la actividad agrícola.

Las muestras se extrajeron en el centro del lecho principal del río, siguiendo normas estandarizadas (Namiesnik y Szefer, 2009). En cada punto de muestreo se recolectaron manualmente $\sim 2 \mathrm{~kg}$ de sedimento, utilizando una pala desde la superficie hasta aproximadamente $15 \mathrm{~cm}$ de profundidad; se tuvo cuidado de no remover el material fino. Los sedimentos fueron depositados en bolsas debidamente etiquetadas hasta su traslado al laboratorio. Se utilizaron materiales plásticos de manera de no contaminar las propiedades magnéticas.

En laboratorio, las muestras de sedimentos fueron secadas a temperatura ambiente y cribadas con tamices Zonytest, certificados por la norma IRAM 1501, con el fin de obtener una muestra Total (T) y separar el material en cinco fracciones granulométricas: fracción I $(>500 \mu \mathrm{m})$, fracción II (entre 500 y $250 \mu \mathrm{m})$, fracción III (entre 250 y $125 \mu \mathrm{m})$, fracción IV (entre 125 y $62.5 \mu \mathrm{m})$ y fracción $\mathrm{V}(<62.5 \mu \mathrm{m})$, las cuales fueron utilizadas para posteriores determinaciones geoquímicas y/o magnéticas.

Además, se realizó un fraccionamiento a través de una extracción secuencial, mediante la cual se eliminan, paso a paso, diferentes fases minerales y los elementos químicos asociados a ellas. Como resultado de esto, en cada paso, se obtienen fracciones residuales diferentes, en las que se realizaron determinaciones magnéticas. El procedimiento de extracción secuencial se llevó a cabo en la fracción $<62.5 \mu \mathrm{m}$ de tamaño de grano (fracción granulométrica V), siguiendo la metodología BCR del Standard Measurements and Testing Program (Ure et al., 1993). Como resultado de este procedimiento, por cada muestra se obtuvieron tres sub-muestras:

-Sub-muestra L-SUQ-X-V-E1: fracción $<62.5 \mu \mathrm{m}$ del sedimento original sin los minerales carbonatados ni los metales asociados con ellos.

- Sub-muestra L-SUQ-X-V-E2: sedimento obtenido de la fracción E1 sin los óxi-hidróxidos de Fe y Mn ni los metales asociados con ellos.

- Sub-muestra L-SUQ-X-V-E3 o residual: fracción constituida por los minerales más resistentes (sedimento obtenido de la fracción E2, sin la materia orgánica, ni sulfuros ni metales asociados).

\section{Determinaciones geoquímicas e índices de calidad}

Se realizaron determinaciones geoquímicas de la fracción $<62.5 \mu \mathrm{m}$, que fueron llevadas a cabo en ActLabs Laboratories (Canadá). Los óxidos mayores se midieron mediante espectrometría de emisión (FUS-ICP), y los elementos traza y elementos de las tierras raras (ETR) mediante espectrometría de masa con plasma inductivamente acoplado (FUS-MS). Las muestras se fusionaron con metaborato/tetraborato de litio y luego se diluyeron con $\mathrm{HNO}_{3}$ al $5 \%$. Se analizó un blanco y 16 materiales de referencia certificados por CANMET, el Instytut Chemii i Techniki Jądrowej y el United States Geological Survey.

Con el fin de evaluar cuantitativamente la contaminación de los sedimentos por metales y metaloides de importancia ambiental, se calcularon diversos índices numéricos ampliamente utilizados en la literatura. El Índice de Geoacumulación $\left(\mathrm{I}_{\text {geo }}\right.$ ) fue definido por Müller (1969) de la siguiente manera:

$$
I_{\text {geo }}=\log _{2}\left(c_{n} / 1.5^{\star} b_{n}\right)
$$

Donde " $c_{n}$ " es la concentración del elemento " $n$ " en la muestra y " $b_{n}$ " es la concentración del elemento " $n$ " en un valor de referencia, por ejemplo, la corteza continental superior (CCS, McLennan 2001). Los valores de $\mathrm{I}_{\text {geo }}$ pueden variar de 0 (ausencia total de contaminación) a 6 (máxima contaminación).

El Factor de Enriquecimiento (FE), definido por Szefer et al. (1998), se basa también en la estandarización de un elemento medido en la muestra contra un elemento de referencia. En este trabajo se utilizó el aluminio como referencia, por su carácter de elemento inmóvil en sistemas exógenos. Así, el FE se define como:

$$
\mathrm{FE}=(\mathrm{Ex} / \mathrm{Al})_{\text {muestra }} /(\mathrm{Ex} / \mathrm{Al})_{\mathrm{CCS}}
$$

Donde $(\mathrm{Ex} / \mathrm{Al})_{\text {muestra }}$ es la relación entre un elemento "Ex" y Al en una muestra y $(\mathrm{Ex} / \mathrm{Al})_{C C S}$ es la relación del elemento "Ex" y Al en la CCS. Sutherland (2000) reconoció cinco categorías de contaminación definidas a través de este índice: $<2$ (enriquecimiento mínimo); entre 2 y 5 (enriquecimiento moderado); entre 5 y 20 (enriquecimiento significativo); entre 20 y 40 (enriquecimiento muy alto) y $>40$ (enriquecimiento extremo).

Por otro lado, Papakostidis et al. (1975) también establecieron diferentes índices de contaminación. Se calculó aquí, el índice de Contaminación Urbana Industrial (C.U.I., expresado en ppm) definido como:

$$
\text { C.U.I. }=[\mathrm{Cu}]+[\mathrm{Pb}]+[\mathrm{Zn}]
$$

Los elementos utilizados en la definición de este índice son considerados indicadores típicos de actividades antrópicas, ya que pueden tener un origen asociado con fuentes de contaminación urbanas e industriales.

\section{Determinación de parámetros magnéticos}

Las determinaciones de los parámetros magnéticos se realizaron en el Laboratorio de Paleomagnetismo y Magnetismo Ambiental del Centro de Investigaciones en Física e Ingeniería del Centro de la Provincia de Buenos Aires (CIFICEN). Se utilizaron técnicas de medición de magnetismo ambiental para la identificación y cuantificación de minerales magnéticos. De cada una de las nueve muestras de sedimentos (Figura 1), se analizaron seis sub-muestras: una muestra total (L-SUQ-X-T) y las cinco fracciones granulométricas determinadas, cuya nomenclatura es: L-SUQ-X-I; L-SUQ-X-II; L-SUQ-X-III; L-SUQ$\mathrm{x}-\mathrm{IV}$ y L-SUQ-X-V, sumando un total de 54 sub-muestras. Además, se analizaron las tres fracciones minerales resultantes de los residuos de las diferentes extracciones secuenciales realizadas de cinco muestras seleccionadas para tal fin (total: 15 sub-muestras). De este modo, las 69 sub-muestras resultantes, fueron pesadas y colocadas en recipientes plásticos de $2.3 \mathrm{~cm}^{3}$.

Las mediciones de susceptibilidad magnética fueron realizadas utilizando un susceptibilímetro MS2 Bartington Instruments Ltd. conectado a un sensor de laboratorio MS2B (en alta frecuencia: $\kappa_{4700 \mathrm{~Hz}}$ y baja frecuencia: $\kappa_{470 \mathrm{~Hz}}$ ). Las mediciones $\kappa_{470 \mathrm{~Hz}}$ fueron normalizadas por volumen (susceptibilidad volumétrica, $\kappa$ ) y masa (susceptibilidad específica, $\chi$ ); además, se calculó el parámetro susceptibilidad magnética dependiente de la frecuencia $\chi_{\mathrm{FD}} \%\left(\frac{\kappa_{470}-\kappa_{4700}}{\kappa_{470}}{ }_{100}\right)$, y el parámetro $\chi_{\mathrm{FD}}\left(=\chi_{470}-\chi_{4700}\right)$.

Por otro lado, para calcular la magnetización remanente anhistérica (MRA) se sometieron las muestras a un campo magnético alterno cuya intensidad varió desde 0 hasta $100 \mathrm{mT}$, y un campo magnético constante $H_{o}$ de $7.95 ; 39.79$ y de $71.62 \mathrm{~A} / \mathrm{m}$. Tales mediciones se realizaron con un dispositivo de MRA parcial adosado a un desmagnetizador 
blindado de campos alternos Molspin Ltd. y un magnetómetro rotativo Minispin, Molspin Ltd. Previamente, las muestras fueron consolidadas con silicato de $\mathrm{Na}$ al $20 \%$ para evitar el movimiento del material. La susceptibilidad anhistérica $\left(\kappa_{\mathrm{MRA}}\right)$ se obtuvo representando los valores corregidos de MRA en función de $H_{o}$ y realizando un ajuste de regresión lineal, donde la pendiente de dicha función es $\kappa_{\mathrm{MRA}}$. Para la susceptibilidad anhistérica específica $\left(\chi_{\mathrm{MRA}}\right)$ se realizó la normalización por peso. Asimismo, se calcularon distintas representaciones y parámetros relacionados, tales como diagrama de King ( $\chi_{\text {MRA }}$ versus $\chi$; King et al., 1982), y el cociente anhistérico $\chi_{\mathrm{MRA}} / \chi$.

\section{RESULTADOS}

\section{Caracterización geoquímica de los sedimentos}

Los sedimentos de cauce del río Suquía tienen granulometría variable, ya que la alta energía del río serrano permite el transporte de sedimentos gruesos (arenas y grava) y finos (suspendidos). Se encontraron sedimentos compuestos principalmente por arenas finas a medianas con mínimas proporciones de guijarros, limos y arcillas. Hasta la estación L-SUQ-5, las fracciones dominantes son las de mayor tamaño de grano: I ( $>500 \mu \mathrm{m})$ y II $(<500 \mu \mathrm{m}$ y $>250 \mu \mathrm{m})$, con porcentajes variables, mayores a $70 \%$. Aguas abajo, en la ciudad de Córdoba, el porcentaje de la fracción más gruesa de los sedimentos disminuye notablemente. La fracción $\mathrm{V}(<62.5 \mu \mathrm{m})$ se analizó geoquímicamente; las concentraciones de los elementos mayores (expresados como porcentaje de óxidos) de los elementos traza y ETR se presentan en la Tabla A1 del suplemento electrónico.

Existen varias propuestas para la caracterización de sedimentos y rocas sedimentarias desde un punto de vista geoquímico, considerando la abundancia de sus componentes mayores. Herron (1988) propuso una clasificación para estimar el tipo de sedimentos en función de su composición química, sobre la base de la clasificación previa de Pettijohn et al. (1972). Ambos esquemas de clasificación están orientados a caracterizar químicamente los grupos de sedimentos previamente clasificados petrográficamente. Con el fin de clasificar químicamente los sedimentos del río Suquía, se utilizó la propuesta de Herron (1988), basada en las relaciones $\mathrm{SiO}_{2} / \mathrm{Al}_{2} \mathrm{O}_{3}$ y $\mathrm{Fe}_{2} \mathrm{O}_{3} / \mathrm{K}_{2} \mathrm{O}$. La primera se empleó como un indicador de madurez mineralógica, ya que los sedimentos mineralógicamente maduros (ricos en cuarzo), tienen una alta relación $\mathrm{SiO}_{2} / \mathrm{Al}_{2} \mathrm{O}_{3}$. Por otra parte, los minerales más estables en sistemas superficiales (p. ej., feldespato potásico, muscovita y cuarzo) poseen bajas relaciones $\mathrm{Fe}_{2} \mathrm{O}_{3} / \mathrm{K}_{2} \mathrm{O}$, mientras que los minerales relativamente más inestables (p. ej., los ferromagnesianos) poseen valores más elevados. Así, la relación $\mathrm{Fe}_{2} \mathrm{O}_{3} / \mathrm{K}_{2} \mathrm{O}$ es una medida de estabilidad mineral. La Figura 2 muestra la clasificación química de los sedimentos de fondo del río Suquía donde se observa que las muestras tienen características químicas comunes a las lutitas, es decir, corresponden a sedimentos con bajas relaciones $\mathrm{SiO}_{2} / \mathrm{Al}_{2} \mathrm{O}_{3}$. Las muestras L-SUQ-4, L-SUQ-5 y L-SUQ-6 poseen concentraciones más elevadas de Fe por lo que, químicamente, representan a lutitas ricas en hierro. Esto refleja la inmadurez de los sedimentos como consecuencia de la cercanía del área de aporte. A modo de comparación, se incluyen en la gráfica sedimentos del lago San Roque (Bertolino et al., 2007), sedimentos del río Los Reartes de áreas prístinas de las Sierras de Córdoba (Pasquini et al., 2004) y un promedio composicional del loess del piedemonte de las Sierras de Córdoba (Pasquini et al., 2017). Como se observa en la Figura 2, los sedimentos del lago y el loess se clasifican como lutitas, mientras que los sedimentos del río Los Reartes corresponden a grauvacas. En todos los casos se trata de sedimentos mineralógicamente inmaduros.

Las concentraciones de elementos traza y ETR en las muestras de sedimento son variables (Tabla A1 del suplemento electrónico). Con el fin de analizar su distribución y variabilidad geoquímica a lo largo del curso medio del río Suquía, las concentraciones absolutas fueron normalizadas al valor promedio de la CCS y se representaron mediante diagramas multielementales. La Figura 3 exhibe los resultados obtenidos, donde se incluye además un promedio composicional para muestras de loess (Pasquini et al., 2017) y la composición normalizada de sedimentos de fondo de ríos de regiones prístinas de las Sierras de Córdoba (Pasquini et al., 2004). En esta figura se observa que la composición de los sedimentos del río Suquía exhibe diferencias importantes con aquella propia de la CCS, del loess y de los sedimentos de áreas prístinas, presentando, en general, mayores concentraciones de elementos traza y ETR. En relación a los elementos mayores, el Si y el Al presentan concentraciones similares a las de la CCS. El Fe, Mn, Ti y P muestran concentraciones más elevadas, mientras que el $\mathrm{Na}$ y el $\mathrm{K}$ se encuentran deprimidos en todas las muestras. El Ca y el Mg muestran mayor variabilidad a lo largo del curso del río. En cuanto a los elementos traza, los que muestran las concentraciones normalizadas más elevadas son As, Y, Zr, Nb, Hf, Th y U. Entre éstos, el As presenta una anomalía positiva que alcanza, en algunos casos, hasta un orden de magnitud superior a los valores de la CCS, mientras que el Sr y Ba muestran concentraciones normalizadas más bajas. Por otro lado, los sedimentos del río Suquía aquí analizados exhiben mayores concentraciones que la de los sedimentos representativos de áreas prístinas y del loess regional.

Con respecto a los ETR (Tabla A1 del suplemento electrónico), los sedimentos del río Suquía presentan, en general, contenidos más altos que la CCS, registrándose concentraciones particularmente elevadas de ETR en la muestra tomada en el campamento Las Bateas (aguas abajo de las canteras en explotación, L-SUQ-4), así como en los sedimentos extraídos en la localidad de río Primero, aguas abajo de la ciudad de Córdoba (muestra L-SUQ-9). En estos dos sitios la concentración total de ETR es de un orden de magnitud superior al contenido de la CCS, mientras que los contenidos más bajos de ETR fueron medidos en la ciudad de Córdoba (muestras L-SUQ-6 y L-SUQ-7).

\section{Índices de contaminación de sedimentos}

Los índices de contaminación de sedimentos calculados en este trabajo se presentan en la Tabla 1. Del análisis de estos índices $\left(\mathrm{I}_{\mathrm{geo}} \mathrm{Y}\right.$ FE) se observa que los sedimentos del río Suquía evidencian grados de contaminación variable, desde no contaminados $\left(\mathrm{I}_{\text {geo }}<0 ; \mathrm{FE}<1\right)$

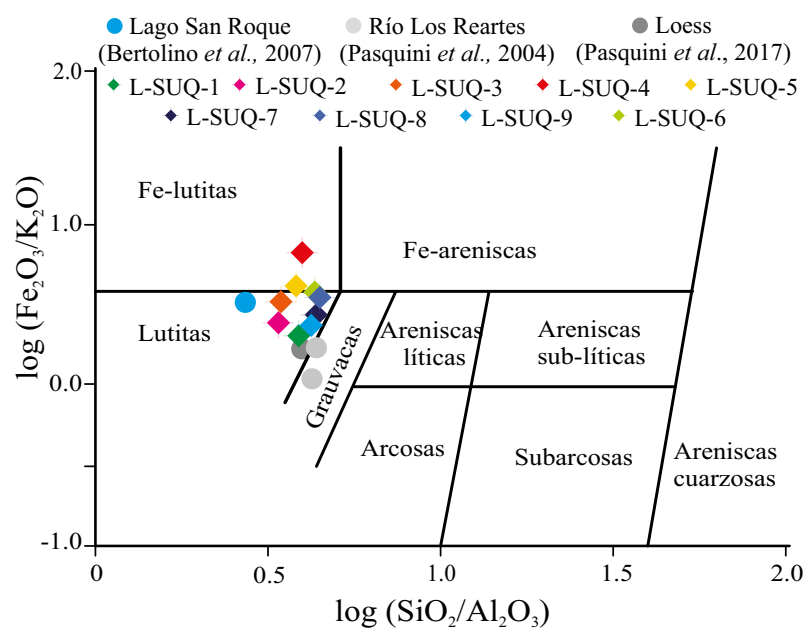

Figura 2. Clasificación química de los sedimentos de fondo del río Suquía según Herron (1988). Se incluyen otros sedimentos a modo de comparación. 


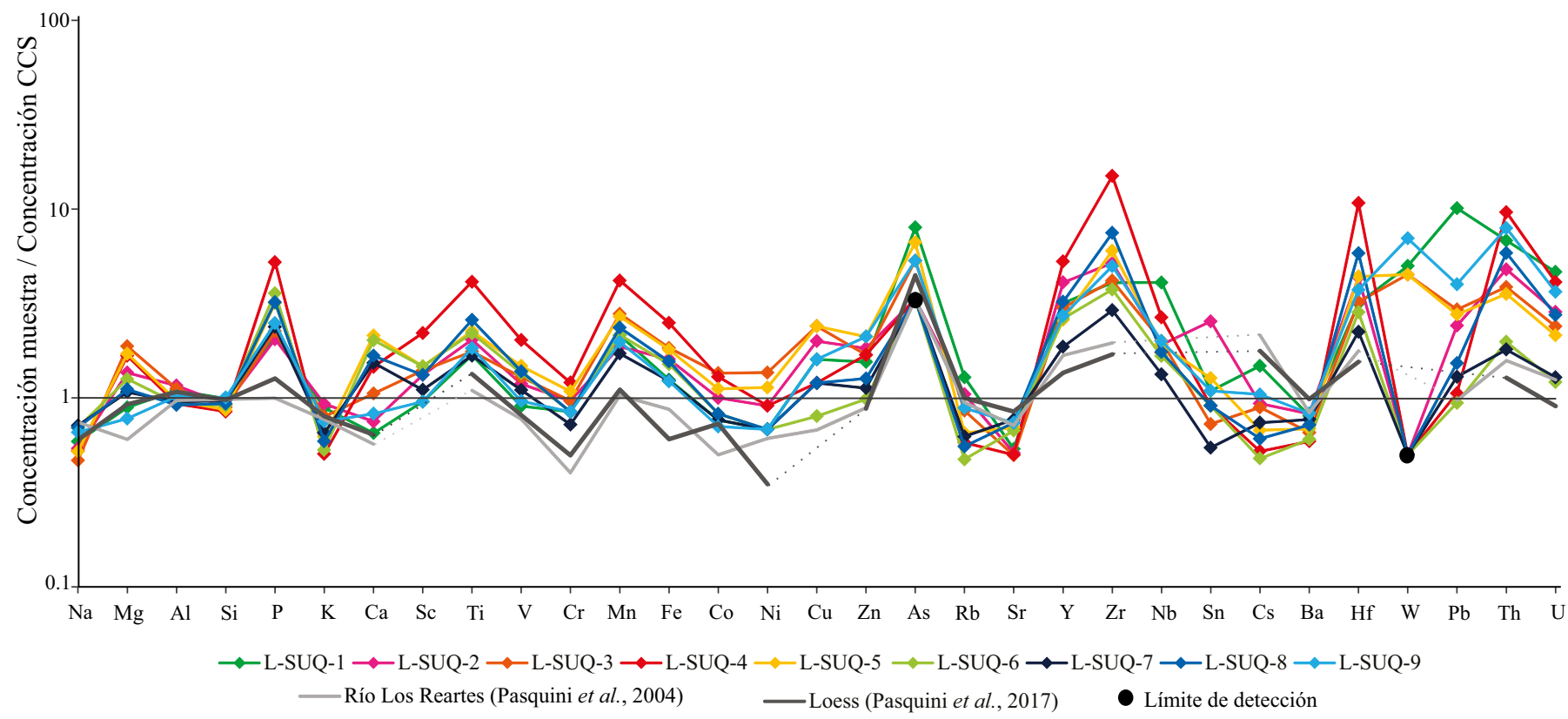

Figura 3. Diagrama multielemental de concentraciones de elementos químicos normalizadas con respecto a la corteza continental superior (CCS, McLennan, 2001). Se incluye también la concentración normalizada de sedimentos del río Los Reartes (Pasquini et al., 2004) y de loess de Córdoba (Pasquini et al., 2017).

hasta contaminación moderada a alta $\left(\mathrm{I}_{\text {geo }}>2 ; \mathrm{FE}>5\right)$. Claramente se observa que en la zona urbana (L-SUQ-6 y L-SUQ-7) existe baja contaminación, excepto para los elementos Hf y Th. Estos elementos, junto con el $\mathrm{U}$ y el $\mathrm{Pb}$ son los que producen mayor contaminación en los sedimentos a lo largo de la cuenca, alcanzando un grado alto y significativo de ambos índices. La zona rural (L-SUQ-9) evidencia un gran número de metales con índices de contaminación altos, especialmente para los elementos antes mencionados. Por otro lado, los elementos $\mathrm{Fe}, \mathrm{Cr}, \mathrm{Cu}, \mathrm{Co}, \mathrm{Ni}$ y $\mathrm{Zn}$ presentan valores de $\mathrm{I}_{\text {geo }}<0$ en su mayoría, lo que indica que los sedimentos no presentan contaminación de estos elementos en el área estudiada.

Por otra parte, las concentraciones de metales en los sedimentos estudiados fueron comparadas con las cantidades recomendadas en la guía interina canadiense de calidad de sedimentos (Interine Sediment Quality Guidelines, ISQGs) desarrolladas por el Canadian Council of Ministers of the Environment (CCME 2001). En la Figura 4 se observan las concentraciones de algunos elementos analizados y sus relaciones con los valores PEL (por sus siglas en inglés probable effect level), por encima del cual se espera que ocurran con frecuencia efectos biológicos adversos. También, se muestran los valores TEL (por sus siglas en inglés threshold effect level), equivalentes al ISQG, por debajo del cual, rara vez se espera que ocurran efectos biológicos adversos. Se observa que el Cr y el $\mathrm{Pb}$ son los únicos elementos que superan el límite PEL en zonas cercanas a las canteras y al lago (L-SUQ-4 y L-SUQ-1 respectivamente). Debido a la concentración de estos elementos, se espera que, con frecuencia, ocurran efectos adversos en el ecosistema. En general, en el resto de la región las concentraciones se encuentran entre los límites guías, pudiendo ocurrir efectos adversos ocasionalmente.

Por último, a partir de la concentración de algunos metales potencialmente tóxicos, se calculó el índice C.U.I. (Tabla 1). Los valores obtenidos varían entre 220 y 320 ppm para el área suburbana, mientras que específicamente en la zona afectada por las canteras, los valores son de $\sim 170$ y 250 ppm. Para el área urbana el índice C.U.I. presenta el menor rango de valores (entre $\sim 100 \mathrm{y} \sim 150 \mathrm{ppm}$ ) y, por

Tabla 1. Índices de Geoacumulación (Igeo), Factores de Enriquecimiento (FE) e Índices de Contaminación Urbana-Industrial (C.U.I.) calculados para los sedimentos del río Suquía.

\begin{tabular}{|c|c|c|c|c|c|c|c|c|c|c|c|c|c|c|c|c|c|c|}
\hline & \multicolumn{2}{|c|}{ L-SUQ-1 } & \multicolumn{2}{|c|}{ L-SUQ-2 } & \multicolumn{2}{|c|}{ L-SUQ-3 } & \multicolumn{2}{|c|}{ L-SUQ-4 } & \multicolumn{2}{|c|}{ L-SUQ-5 } & \multicolumn{2}{|c|}{ L-SUQ-6 } & \multicolumn{2}{|c|}{ L-SUQ-7 } & \multicolumn{2}{|c|}{ L-SUQ-8 } & \multicolumn{2}{|c|}{ L-SUQ-9 } \\
\hline $\mathrm{Fe}$ & -0.44 & 1.01 & -0.08 & 1.22 & 0.02 & 1.36 & 0.61 & 2.46 & 0.08 & 1.64 & -0.11 & 1.49 & -0.41 & 1.18 & -0.05 & 1.58 & -0.40 & 1.11 \\
\hline $\mathrm{Cr}$ & -0.83 & 0.77 & -0.64 & 0.83 & -0.64 & 0.86 & -0.32 & 1.29 & -0.47 & 1.12 & -0.83 & 0.90 & -1.05 & 0.76 & -0.83 & 0.92 & -0.83 & 0.83 \\
\hline $\mathbf{N i}$ & -1.14 & 0.62 & -0.72 & 0.78 & -0.14 & 1.22 & -0.72 & 0.98 & -0.40 & 1.17 & -1.14 & 0.73 & -1.14 & 0.72 & -1.14 & 0.74 & -1.14 & 0.67 \\
\hline $\mathrm{Cu}$ & 0.09 & 1.46 & 0.42 & 1.72 & 0.68 & 2.14 & -0.32 & 1.29 & 0.68 & 2.48 & -0.91 & 0.86 & -0.32 & 1.26 & -0.32 & 1.31 & 0.09 & 1.57 \\
\hline Zn & 0.05 & 1.41 & 0.29 & 1.58 & 0.17 & 1.51 & 0.17 & 1.82 & 0.49 & 2.18 & -0.61 & 1.05 & -0.41 & 1.18 & -0.24 & 1.38 & 0.49 & 2.07 \\
\hline $\mathbf{P b}$ & 2.75 & 9.23 & 0.69 & 2.08 & 0.97 & 2.63 & -0.50 & 1.14 & 0.88 & 2.85 & -0.67 & 1.01 & -0.21 & 1.36 & 0.03 & 1.66 & 1.42 & 3.92 \\
\hline Th & 2.18 & 6.22 & 1.68 & 4.13 & 1.37 & 3.46 & 2.68 & 10.34 & 1.24 & 3.67 & 0.40 & 2.12 & 0.27 & 1.89 & 1.97 & 6.37 & 2.40 & 7.78 \\
\hline $\mathbf{U}$ & 1.63 & 4.24 & 0.93 & 2.46 & 0.67 & 2.14 & 1.45 & 4.41 & 0.51 & 2.21 & -0.30 & 1.30 & -0.22 & 1.35 & 0.87 & 2.99 & 1.28 & 3.57 \\
\hline C.U.I & \multicolumn{2}{|c|}{322} & \multicolumn{2}{|c|}{221} & \multicolumn{2}{|c|}{230} & \multicolumn{2}{|c|}{168} & \multicolumn{2}{|c|}{257} & \multicolumn{2}{|c|}{106} & \multicolumn{2}{|c|}{132} & \multicolumn{2}{|c|}{146} & \multicolumn{2}{|c|}{258} \\
\hline
\end{tabular}




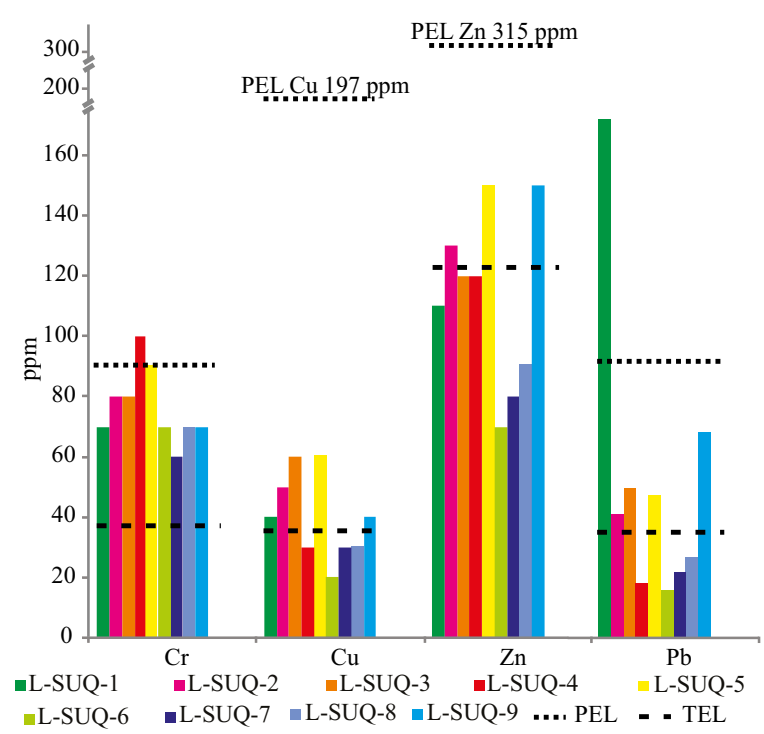

Figura 4. Concentración de metales en los sedimentos del río Suquía y relación con los valores PEL y TEL (CCME, 2001) definidos para estos elementos.

último, en la zona rural vuelve a mostrar valores altos ( 250 ppm). El orden de contaminación observado a partir del C.U.I. es el siguiente: L-SUQ-1 > L-SUQ-9 > L-SUQ-5 > L-SUQ-3 > L-SUQ-2 > L-SUQ-4 > L-SUQ-8 > L-SUQ-7 > L-SUQ-6.

\section{Propiedades magnéticas}

Los parámetros magnéticos de los sedimentos del río Suquía se analizaron con el fin de determinar el grado de contaminación a través de la señal magnética. Se tomaron en cuenta, por un lado, las diferentes fracciones granulométricas y por otro, las fracciones residuales resultantes de la extracción secuencial.

En la Figura 5 se presentan, para cada sitio de muestreo, los resultados de los parámetros dependientes de la concentración magnética, susceptibilidad magnética específica (5a) y MRA (5c), correspondientes a las fracciones: Total, I, II, III, IV y V, así como el porcentaje de las fracciones granulométricas (5b). Los valores máximos de $\chi$ en muestra total $\left(\chi=540.7 \times 10^{-8} \mathrm{~m}^{3} \mathrm{~kg}^{-1}\right.$, Figura 5a) se observan en la región cercana a la cantera (L-SUQ-4). Aguas abajo, se observa una disminución de los valores de $\chi\left(\chi \sim 35.0 \times 10^{-8} \mathrm{~m}^{3} \mathrm{~kg}^{-1}\right)$, para luego volver a aumentar en la ciudad de Córdoba (L-SUQ-8, $\chi \sim 120.0 \times 10^{-8} \mathrm{~m}^{3} \mathrm{~kg}^{-1}$ ). Un comportamiento similar se observa en las diferentes fracciones granulométricas (Figura 5a), excepto en algunas fracciones, aunque su contribución (concentración en \%) a la muestra total no resulta significativa (Figura 5b). Los valores obtenidos en la muestra L-SUQ-5 pueden estar influenciados por la muestra L-SUQ-4 debido a su proximidad a la cantera. Se puede observar que la $\chi$ obtenida para la muestra total es similar a la de las fracciones $\mathrm{V}(<62.5 \mu \mathrm{m})$ de las muestras L-SUQ-4 y L-SUQ-5. Por otro lado, aguas abajo, en el área urbana (L-SUQ-6, L-SUQ-7 y L-SUQ-8) y rural (L-SUQ-9), los valores de $\chi$ de la fracción $\mathrm{V}$ son mayores (duplican/triplican sus valores, Tabla A2 del suplemento electrónico) respecto a la de muestra total. Se observa que la fracción V tiene una concentración menor $(0.4-0.6 \%)$ en los sitios L-SUQ- 4 y L-SUQ-5 respecto a las restantes fracciones granulométricas, así como respecto a los sitios aguas abajo. Las muestras con mayor porcentaje de la fracción V son L-SUQ-6 (15.4 \%) y L-SUQ-9 (4.1 \%).

A partir del parámetro $\chi_{\mathrm{FD}} \%$ (Tabla A2) se puede observar que en la zona de estudio hay escasa o nula presencia de granos superparamagnéticos (SP), debido a que los valores de $\chi_{\mathrm{FD}} \%$ son menores al $2 \%$, excepto para las zonas suburbanas (L-SUQ-1, L-SUQ-2, L-SUQ-3) y rural (L-SUQ-9). En las fracciones finas de la muestra L-SUQ-6 hay presencia de granos SP (granos magnéticos ultrafinos $<0.03 \mu \mathrm{m}$ ) mezclados con granos más grandes $\left(\chi_{\mathrm{FD}} \% \sim 2-10 \%\right)$. Asimismo, a partir del parámetro $\chi_{\mathrm{FD}}$ es posible semi-cuantificar la concentración de granos ferrimagnéticos SP y determinar si éstos son dominantes (Dearing et al., 1996). De acuerdo a los resultados listados en la Tabla A2, los valores de $\chi_{\mathrm{FD}}$ varían entre 0.1 y $8.4 \times 10^{-8} \mathrm{~m}^{3} \mathrm{~kg}^{-1}$, lo cual representa una mínima contribución en comparación al resto de los granos magnéticos. Las muestras totales presentan sus mayores valores de $\chi_{\mathrm{FD}}\left(4.7-6.4 \times 10^{-8} \mathrm{~m}^{3} \mathrm{~kg}^{-1}\right)$ aguas abajo del lago (L-SUQ-2 y L-SUQ-3), mientras en la zona urbana (L-SUQ-5, L-SUQ-6, L-SUQ-7, y L-SUQ-8) los valores son menores y similares entre sí, siendo $\chi_{\mathrm{FD}}=0.8-1.0 \times 10^{-8} \mathrm{~m}^{3} \mathrm{~kg}^{-1}$.

La MRA es un parámetro que depende de la concentración de minerales ferrimagnéticos del tipo magnetita (Chaparro et al., 2014). Los valores de MRA obtenidos en toda la región varían en un amplio rango, desde 24.7 hasta $632.1 \times 10^{-6} \mathrm{Am}^{2} \mathrm{~kg}^{-1}$ (Tabla A2, Figura 5c). Un análisis multivariado de los parámetros magnéticos correspondientes a cada grupo de muestras (muestra total y fracciones granulométricas), arrojó una correlación estadísticamente significativa $(\rho<0.01)$ entre MRA y $\chi$; no obstante, el coeficiente de Pearson es mayor para las muestras totales $(r=0.95)$ que para todas las muestras $(r=0.72)$. Esto indica que la señal magnética es dominada por minerales ferrimagnéticos en las muestras totales, pero no así en todas las fracciones granulométricas. En las Figuras 5 a y 5 c se observa que, en las muestras totales, los parámetros dependientes de la concentración magnética presentan un comportamiento similar, con valores máximos en la región de la cantera (L-SUQ-4, MRA $=351.2 \times 10^{-6} \mathrm{Am}^{2} \mathrm{~kg}^{-1}$ ). No obstante, la fracción $\mathrm{V}$ presenta, en toda la región, valores mayores de MRA respecto a la muestra total, lo cual indica que existe mayor concentración de minerales ferrimagnéticos en la fracción granulométrica más fina. Se puede observar que la fracción $\mathrm{V}$ tiene los mayores valores de MRA en zona urbana (L-SUQ-7; L-SUQ-8) y en la zona rural (L-SUQ-9).

Mediante el gráfico de King (Figura 6), se realizaron estimaciones cuantitativas de tamaños de granos magnéticos, representando $\chi_{\text {MRA }}$ en función de $\chi$, junto con rectas de calibración de distintos tamaños de granos de magnetita. Los resultados obtenidos indican que los granos magnéticos varían desde 0.2 hasta $20 \mu \mathrm{m}$. Para las muestras totales, se observa, por un lado, la presencia de granos magnéticos más finos $(1-5 \mu \mathrm{m})$ en la zona urbana (L-SUQ-6, L-SUQ-7 y L-SUQ-8) y, por otro lado, granos magnéticos de mayor tamaño $(5-20 \mu \mathrm{m})$ en la cantera (L-SUQ-4) y sus alrededores (L-SUQ-3 y L-SUQ-5). En general, se observan granos magnéticos menores a $1 \mu \mathrm{m}$ para la fracción más fina V (Figura 6), excepto para la zona de la cantera (L-SUQ-4), que se encuentra en el rango de $5 \mu \mathrm{m}$.

En la Figura 7 se representa la relación entre parámetros dependientes del tamaño de grano magnético (cociente anhistérico $\chi_{\mathrm{MRA}} / \chi$ ) y la concentración magnética $(\chi)$. Las correlaciones entre estos parámetros son estadísticamente significativas, aunque son mayores para la fracción más fina $\mathrm{V}(r=-0.81 ; \rho<0.01)$. Si bien no se observaron valores de $\chi_{\text {MRA }} / \chi>5$ indicativos de minerales ferrimagnéticos muy finos $<0.1 \mu \mathrm{m}$ (Peters y Dekkers, 2003); los valores más altos de $\chi_{\mathrm{MRA}} / \chi$ (esto es, granos magnéticos más finos) en las muestras totales, se observan principalmente en zonas urbanas (L-SUQ-6, L-SUQ-7 y L-SUQ-8, Figura 7). La fracción granulométrica $\mathrm{V}$ para cada sitio presenta, en general, valores mayores respecto a la muestra total.

Con el fin de determinar partículas contaminadas adsorbidas en fracciones biodisponibles en los sedimentos, se analizaron también las propiedades magnéticas (p. ej., MRA y $\chi_{\mathrm{MRA}} \chi \chi$ ) de las fracciones residuales luego de las tres extracciones secuenciales (E1, E2 y E3). Los resultados indican que, en general, las variables magnéticas no cambian apreciablemente (Figura 8a, 8b). Al analizar el tamaño de 

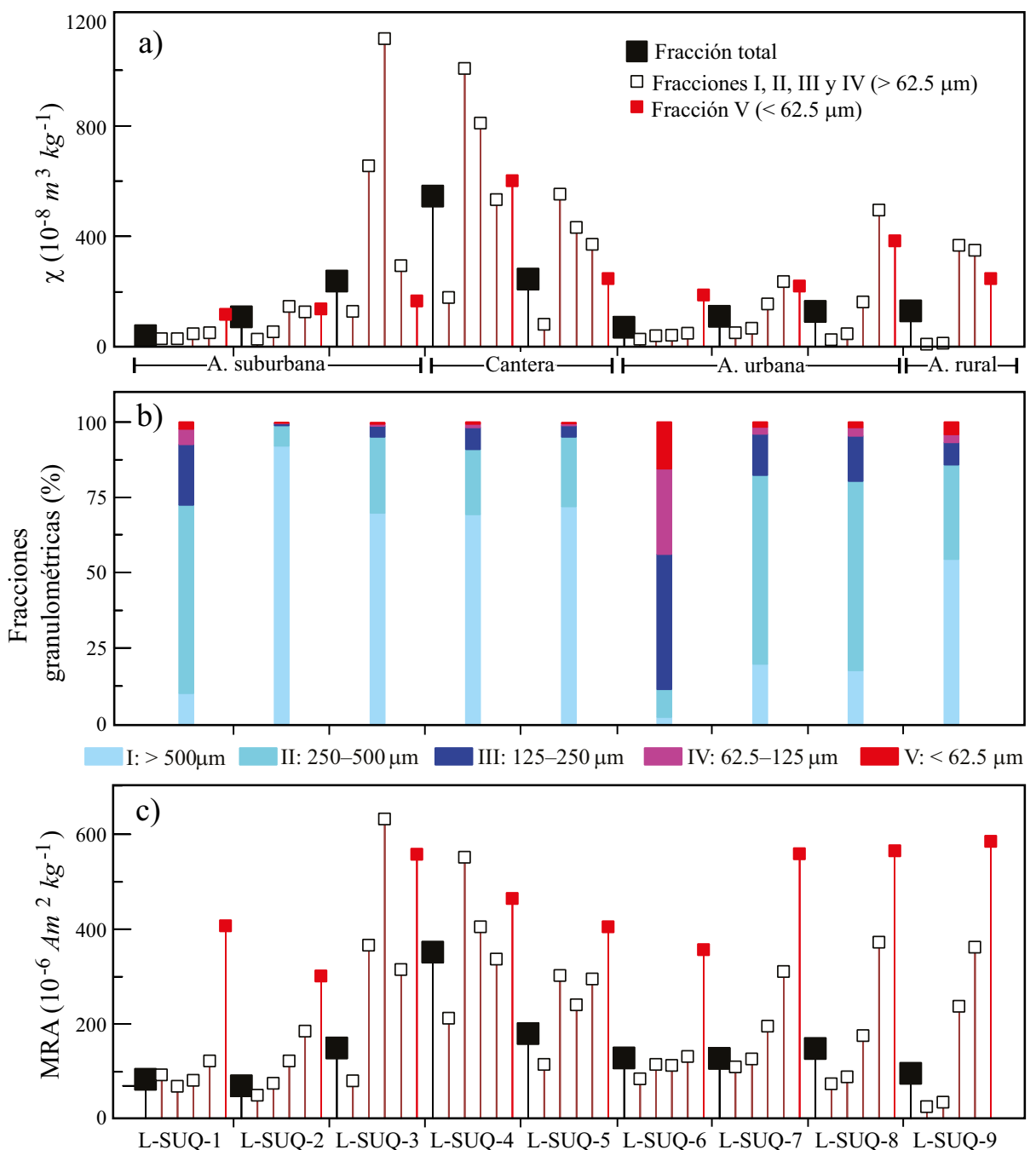

Figura 5. Representación de: a) la susceptibilidad magnética específica, b) porcentaje de las fracciones granulométricas y c) MRA para cada sitio de muestreo. Se detalla cuáles son los sitios en áreas urbanas y suburbanas.

grano magnético, se puede observar que respecto a la muestra inicial (Fracción $\mathrm{V},<62.5 \mu \mathrm{m}$ ) los resultados son similares en las posteriores fracciones residuales (Figura $8 \mathrm{~b}$ ). Por su parte, la concentración de minerales ferrimagnéticos presenta una disminución a través de la secuencia de extracciones en L-SUQ-1, L-SUQ-4 y L-SUQ-8 y un leve incremento en las muestras L-SUQ-5 y L-SUQ-9 (Figura 8a).

\section{DISCUSIÓN}

Los sedimentos de fondo del río Suquía presentan, en términos generales, concentraciones más elevadas de metales y metaloides que sedimentos de ríos de áreas prístinas de las Sierras de Córdoba. Los procesos que dan origen a las características geoquímicas y magnéticas de estos sedimentos se diferencian espacialmente, a lo largo de la cuenca y se deben a factores tanto naturales como antrópicos. Además, la comparación con datos previamente publicados (Harguinteguy et al., 2014; Monferrán, 2018) permite advertir que las concentraciones geoquímicas se han modificado durante la última década.

Los parámetros magnéticos dependientes de la concentración magnética ( $\chi$ y MRA) de los sedimentos del río Suquía muestran una

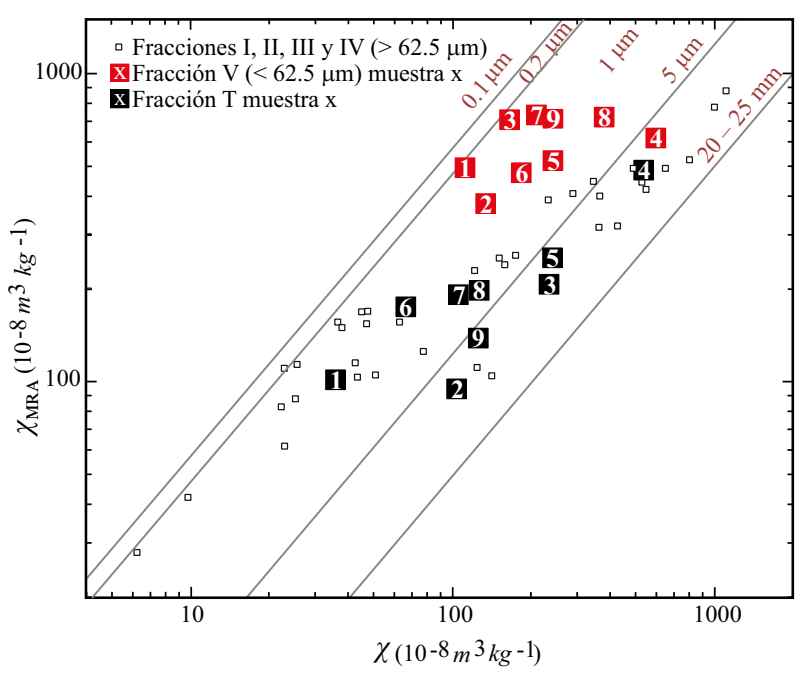

Figura 6. Determinaciones cuantitativas de tamaños de granos magnéticos. Representación de $\chi_{\mathrm{MRA}}$ en función de $\chi$, junto con rectas de calibración de distintos tamaños de grano de magnetita (Gráfico de King). 


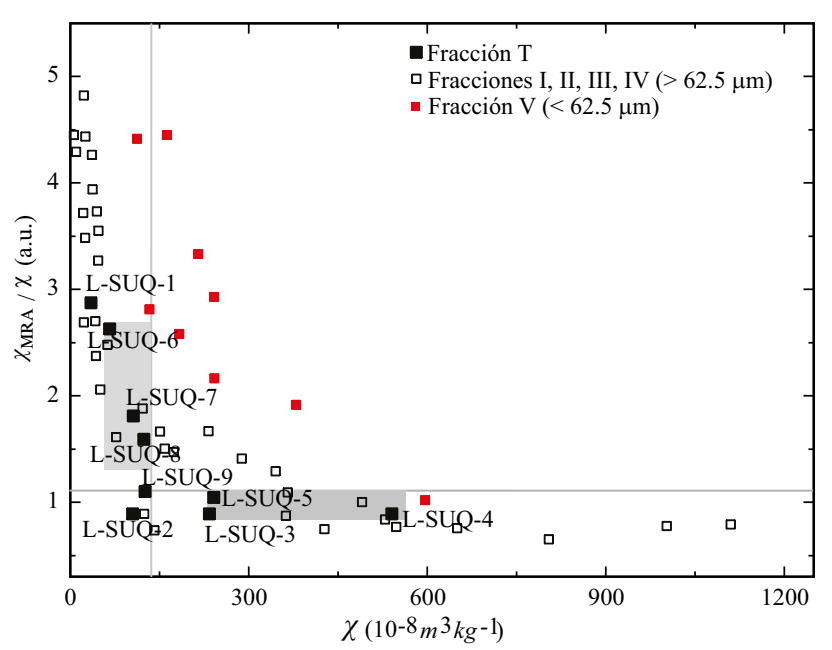

Figura 7. Gráfica de parámetros dependientes del tamaño de grano magnético (cociente anhistérico $\chi_{\mathrm{MRA}} / \chi$ ) y de la concentración magnética $(\chi)$. La relación entre los parámetros es inversa, lo cual indica que la mayor concentración magnética se asocia a tamaños de granos mayores.

amplia variación a lo largo del río que contrasta la zona de canteras (máximos valores) con las áreas urbanas y rurales.

Del análisis espacial se desprende que aguas abajo del lago San Roque, región suburbana, la alta concentración de metales determinada deriva principalmente de las rocas metamórficas del área de aporte, evidenciando un origen natural. En este sector los diferentes índices de contaminación calculados indican un grado moderado a alto para algunos elementos ( $\mathrm{Pb}$ y Th, y en menor medida $\mathrm{U}$ y Hf). La presencia en esta región de ortogneis piroxénico y tonalítico, como también milonita, gabro y mármol, deja claramente su impronta en los sedimentos del río Suquía.

En la zona de canteras en explotación, se evidencia un aumento significativo en las concentraciones de metales pesados como $\mathrm{Fe}, \mathrm{Mn}$, $\mathrm{Mg}$, Ti, P, V, Cr, Co, Zr, Hf, Th, U y ETR. Este incremento viene acompañado de un aumento de los valores en los parámetros magnéticos dependientes de la concentración, y minerales ferrimagnéticos de granos de mayor tamaño (5-20 $\mu \mathrm{m})$, así como también un aumento de los índices de contaminación. La explotación de las canteras (principalmente rocas de aplicación: granito, pegmatita y mármol) genera un incremento en la superficie de las rocas expuestas a la meteorización, ya que las mismas son extraídas mediante métodos de voladura y, por lo tanto, quedan partidas en fragmentos más pequeños. Además, los procesos posteriores de rompimiento para reducir aún más el tamaño de los bloques a extraer, también aumentan el número de superficies que atacan la meteorización y la erosión, facilitando, de esta manera, la incorporación de los elementos antes mencionados a los sedimentos del rio. La titanita, magnetita, titanomagnetita e ilmenita, todos óxidos de Fe y accesorios comunes presentes en las rocas de la región, pueden incluir Mn y Ti por sustitución en sus estructuras. El circón también es un accesorio común que puede incluir $\mathrm{Hf}, \mathrm{U}$ y Th. La biotita presente en el gneis del grupo El Diquecito es rica en Fe (Locati et al., 2009). Así mismo, los clinopiroxenos, ortopiroxenos y hornblenda son minerales ricos en Fe, Mn y Mg. Como se ha discutido, los máximos valores de $\chi$ y MRA (mayor concentración magnética) se asocian a tamaños de granos magnéticos mayores. Una correlación positiva entre el parámetro $\chi \mathrm{y}$ los elementos Fe, Mn, Ti, P, Sc, V, Zr, ETR, y Hf ( $r$ entre 0.75 y 0.93 ; $\rho<0.01)$ refuerza el origen litológico de estas características, potenciado por las actividades de explotación de la cantera.

En la zona urbana la concentración de metales y metaloides disminuye significativamente y, por lo tanto, los diferentes índices de contaminación alcanzan valores mínimos. Los únicos elementos que presentan índices de contaminación moderada son Hf y Th. La disminución de las concentraciones de metales pesados en la ciudad de Córdoba puede ser el resultado de la presencia de la cubierta fluvial-eólica, cuyos sedimentos presentan una menor concentración de metales que las rocas del basamento. Esto queda evidenciado en los diagramas multielementales donde las concentraciones relativas de metales en el loess son significativamente menores.

Aguas abajo, en el área rural de la cuenca, las actividades agrícolas también generan un impacto en los sedimentos de fondo del río, incrementando la concentración de metales. En esta región, los índices de contaminación van de moderados a significativos en Th, Pb, Hf, $\mathrm{U}$ y $\mathrm{Zn}$. Algunos de estos metales pueden derivar de los fungicidas y fertilizantes utilizados en la agricultura (Otero et al., 2005; Vargas, 2010). En esta región se observa, además, un aumento significativo de ETR. Los fertilizantes fosfatados son una fuente antropogénica relevante de ETR, ya que generalmente se producen a partir de fosfatos naturales ricos en ETR debido a la alta afinidad entre ellos (Laveuf y Cornu, 2009). Así, es posible que las elevadas concentraciones de ETR medidas en los sedimentos de la zona rural estén controladas por las actividades antrópicas en esa región. Sin embargo, los parámetros magnéticos medidos en las diferentes fracciones residuales (E1 - Fracción intercambiable, E2 - Fracción reducible y E3 - Fracción oxidable) a lo largo de toda la cuenca, indican, en general, que los minerales ferrimagnéticos no se encuentran asociados a ninguna fase mineral en particular. De esto se desprende que la señal magnética de estos sedimentos está controlada por los minerales residuales o los de origen antrópico. Tal resultado puede interpretarse en términos de una muy baja cantidad de minerales ferrimagnéticos extraíbles y/o una alta resistencia de los óxidos de hierro ferrimagnéticos, tanto los de origen natural (rocas) como los de origen antrópico.

La correlación estadísticamente significativa $(\rho<0.01)$ entre $\chi$ y MRA indica que la señal magnética es dominada por minerales ferrimagnéticos del tipo magnetita. Aunque la concentración porcentual de las fracciones $\mathrm{V}(<62.5 \mu \mathrm{m})$ varía apreciablemente a lo largo del río, tales minerales ferrimagnéticos se encuentran en mayor concentración en esta fracción y se caracterizan por tamaños de grano más finos. El análisis multivariado entre parámetros dependientes del tamaño de grano magnético $\left(\chi_{\mathrm{MRA}} \chi\right)$ y de la concentración magnética $(\chi)$ muestra una correlación estadísticamente significativa e inversa, lo cual indica
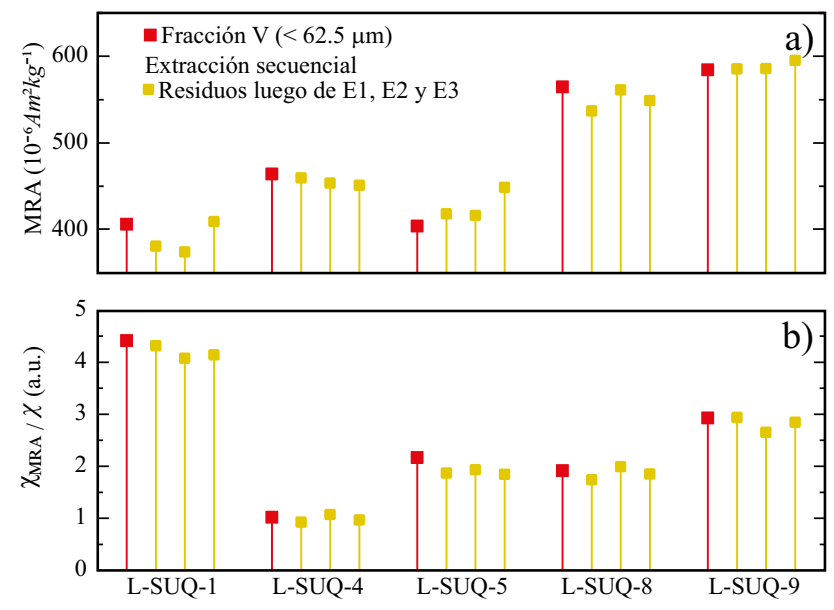

Figura 8. Propiedades magnéticas de la fracción $\mathrm{V}(<63 \mu \mathrm{m})$ y las fracciones residuales luego de las tres extracciones secuenciales (E1, E2 y E3). 
Tabla 2. Concentraciones ( $\mathrm{ppm}$ ) de metales en la fracción $<62.5 \mu \mathrm{m}$, en la cuenca media del río Suquía y en la localidad de La Calera. ${ }^{2}$ Harguinteguy et al. (2014),

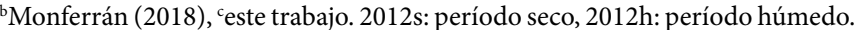

\begin{tabular}{cccccc}
\hline & a2006 & a2009 & b2012s & b2012h & '2014 \\
\hline Mínimo & & & & & \\
$\mathrm{Cr}$ & - & - & 21 & 28 & 60 \\
$\mathrm{Cu}$ & 5.46 & 7.73 & 18 & 13 & 20 \\
$\mathrm{Zn}$ & 46.8 & 32.2 & 27 & 38 & 70 \\
$\mathrm{~Pb}$ & 14.1 & 8.92 & 10 & 9 & 16 \\
$\mathrm{Ni}$ & 9.26 & 7.59 & 10.4 & 11 & 30 \\
$\mathrm{Máximo}$ & & & & & \\
$\mathrm{Cr}$ & - & - & 26 & 31 & 100 \\
$\mathrm{Cu}$ & 6.66 & 12.55 & 30 & 44 & 60 \\
$\mathrm{Zn}$ & 71.6 & 58.4 & 78 & 126 & 150 \\
$\mathrm{~Pb}$ & 18.93 & 20.87 & 34 & 39 & 172 \\
$\mathrm{Ni}$ & 20.26 & 12.91 & 12.1 & 13.8 & 60 \\
$\mathrm{La}$ Calera & & & & & \\
$\mathrm{Cu}$ & 5.69 & 7.73 & 18 & 13 & 60 \\
$\mathrm{Zn}$ & 46.8 & 44.8 & 27 & 38 & 150 \\
$\mathrm{~Pb}$ & 14.1 & 13.42 & 10 & 9 & 47 \\
$\mathrm{Ni}$ & 9.26 & 8.83 & 10.4 & 11 & 50 \\
\hline & & & & &
\end{tabular}

que la mayor concentración de minerales ferrimagnéticos se asocia a tamaños de granos mayores. Los valores más altos de $\chi_{\mathrm{MRA}} / \chi$ se pueden observar principalmente en zonas urbanas. En este caso, el análisis conjunto del cociente anhistérico y el parámetro $\chi$ representado en la Figura 7, es de gran utilidad para diferenciar claramente la zona urbana (L-SUQ-6, L-SUQ-7 y L-SUQ-8) de la cantera (L-SUQ-4), sus alrededores (L-SUQ-3 y L-SUQ-5) y la zona suburbana (L-SUQ-1, L-SUQ-2) y zona rural (L-SUQ-9).

La evolución temporal en las concentraciones de metales en los sedimentos del río Suquía se analizó comparando los datos aquí presentados con aquellos reportados previamente (Harguinteguy et al., 2014; Monferrán, 2018). Nuestra investigación se realizó mediante técnicas analíticas comparables y en la misma fracción granulométrica. Se observó un incremento general en las concentraciones de metales a través del tiempo, particularmente en la localidad de La Calera (Tabla 2). Esto indica que, efectivamente, los sedimentos del río Suquía están actuando como trampas de concentración de elementos potencialmente tóxicos. Sin embargo, el nivel de contaminación de estos sedimentos no es comparable con sistemas altamente contaminados como, por ejemplo, la cuenca Riachuelo-La Matanza, (Brigden et al., 2000; Malpartida, 2003; Puig y Olguín, 2006). En dicha cuenca, los valores obtenidos de $\mathrm{I}_{\text {geo }}$ variaron entre $\sim 3 \mathrm{y} \sim 5$, evidenciando una contaminación alta a extrema en $\mathrm{Pb}, \mathrm{Zn}, \mathrm{Cu}$, Co y $\mathrm{Cr}$; mientras que en el río Suquía, estos metales presentan $\mathrm{I}_{\text {geo }}<3$. Así, aunque en la actualidad los índices de contaminación no presentan señales de un impacto antrópico elevado (desde un enfoque inorgánico), en algunos años el escenario podría empeorar.

\section{CONCLUSIONES}

En este estudio fue posible demostrar la potencialidad del uso combinado de herramientas geoquímicas y magnéticas para evaluar el grado de contaminación de un sistema natural con alteración antrópica. La aplicación de estas herramientas al sistema hídrico del río Suquía en la región central de Argentina, permitió identificar regiones de la cuenca con altos niveles de metales en los sedimentos asociados a fuentes naturales. De la misma forma, se identificaron regiones donde los niveles de contaminación por metales pesados parecen más controlados por factores antrópicos. Además, los parámetros magnéticos, en particular, señalan que existen zonas que se encuentran más afectadas por las actividades antrópicas, ya que hay una acumulación de minerales magnéticos en las mismas. En este río, que discurre desde regiones serranas y atraviesa una ciudad densamente poblada, diferentes procesos determinan las características geoquímicas y las propiedades magnéticas de sus sedimentos de fondo. La región suburbana y aguas abajo de ésta, donde se explota mármol y rocas de aplicación, se caracteriza por una concentración elevada de metales, así como de minerales ferrimagnéticos asociados a una fuente natural y potenciada por actividades antrópicas. En la región urbana se determinaron, en general, concentraciones más bajas de estos parámetros debido a un cambio en las características geológicas (presencia de una cubierta de loess) y a cambios en el uso del suelo (urbanización). Aguas abajo de la región más urbanizada, en la zona rural, niveles altos de contaminación de metales (incluidos ETR) se asocian a actividades agrícolas (p. ej. uso de fertilizantes).

Resulta importante señalar que la relación entre variables magnéticas y geoquímicas valida el uso de la metodología de monitoreo magnético como una herramienta complementaria para la evaluación de ambientes con influencia antrópica. Su ventaja reside en el hecho de que la toma de mediciones no presenta un costo significativo, ya que la muestra no precisa tratamiento químico previo o preparación especial. Además, la metodología puede ser usada para evaluar, en una primera aproximación, la contaminación de forma económica y rápida sobre áreas con retículas de distinta densidad.

\section{AGRADECIMIENTOS}

Los autores agradecen a la Universidad Nacional de Córdoba (UNC), Universidad Nacional del Centro de la Provincia de Buenos Aires (UNCPBA), CONICET y a la Agencia Nacional de Promoción Científica y Tecnológica (ANPCyT) por el apoyo financiero. Esta contribución se desarrolló en el marco de los proyectos PICT-2012 No 0275, SECyT-UNC (05/I711 y 05/I754) y PIP No 112-200801-03160. Así mismo, agradecemos a dos revisores anónimos por sus valiosos aportes que han contribuido a mejorar sustancialmente este trabajo.

\section{MATERIAL SUPLEMENTARIO}

Las Tablas A1 y A2, están disponibles como suplemento electrónico en el portal web de la Revista, en la tabla de contenido de este número.

\section{REFERENCIAS}

Bertolino, S., Zimmermann, U., Sattler, F., 2007, Mineralogy and geochemistry of bottom sediments from water reservoirs in the vicinity of Córdoba, Argentina: environmental and health constraints: Applied Clay Science, 36(1), 206-220.

Brigden, K., Labunska, I., Stringer, R., Johnston, P., Santillo, D., Ashton, J., 2000, Contaminantes orgánicos y metales pesados en vertidos y sedimentos del Riachuelo, Argentina: Exeter, Reino Unido, Laboratorios de Investigación de Greenpeace, Departamento de Ciencias Biológicas, Universidad de Exeter, Nota técnica, $50 \mathrm{pp}$.

Campodonico, V.A., Martínez, J.O., Verdecchia, S.O., Pasquini, A.I., Depetris, P.J., 2014, Weathering assessment in the Achala Batholith of the Sierra de Comechingones, Córdoba, central Argentina. I: Granite-regolith fractionation: Catena, 123, 121-134. 
Carson, M., Kirkby, M., 1972, Hillslope form and process: Science, 178, 1083-1084.

CCME (Canadian Council of Ministers of the Environment), 2001, Canadian Sediment Quality Guidelines for the Protection of Aquatic Life, Water Quality Index 1.0, Technical Report: Winnipeg, MB, Canada.

Chaparro, M.A.E., Gogorza, C.S.G., Chaparro, M.A.E., Irurzun, M.A., Sinito, A.M., 2006, Review of magnetism and heavy metal pollution studies of various environments in Argentina: Earth, Planets and Space, 58(10), 1411-1422.

Chaparro, M.A.E., Chaparro, M.A.E., Rajkumar, P., Ramasamy, V., Sinito, A.M., 2012, Magnetic parameters, trace elements, and multivariate statistical studies of river sediments from southeastern India: a case study from the Vellar River: Environmental Earth Science, 63(2), 297-310.

Chaparro, M.A.E., Gargiulo, M., Irurzun, M.A., Chaparro, M.A.E., Lecomte, K.L., Böhnel, H.N., Córdoba, F.E., Vignoni, P.A., Czalbowski, N.T.M., Lirio, J.M., Nowaczyk, N.R., Sinito, A.M., 2014, El uso de parámetros magnéticos en estudios paleolimnológicos en Antártida: Latin American Journal of Sedimentology and Basin Analysis, 21(2), 77-96.

Chaparro, M.A.E., Krishnamoorthy, N., Chaparro, M.A.E., Lecomte, K.L., Mullainathan, S., Mehra, R., Sinito, A.M., 2015, Magnetic, chemical and radionuclide studies of river sediments and their variation with different physiographic regions of Bharathapuzha river, southwestern India: Studia Geophysica et Geodaetica, 59(3), 438-460.

Chaparro, M.A.E., Chaparro, M.A.E., Córdoba, F.E., Lecomte, K.L., Gargiulo, J.D.M., Barrios, A.M., Urán, G., Czalbowski, N.T.M., Lavat, A., Böhnel, H., 2017a, Sedimentary analysis and magnetic properties of Lake Anónima, Vega Island: Antarctic Science, 1(16), 429-444.

Chaparro, M.A.E., Suresh, G., Chaparro, M.A.E., Ramasamy, V., Sundarrajan, M., 2017b, Magnetic assessment and pollution status of beach sediments from Kerala coast (southwestern India): Marine Pollution Bulletin, 117(1-2), 171-177.

Dasso, C.M., Piovano, E.L., Pasquini, A.I., Córdoba, F.E., Lecomte, K.L., Guerra, L., Campodonico, V.A., 2014, Recursos Hídricos Superficiales, en Córdoba-Martino, R.D., Guereschi, A.B. (eds.), Relatorio del XIX Congreso Geológico Argentino: Geología y Recursos Naturales de la Provincia de Córdoba, Asociación Geológica Argentina, Córdoba, 1209-1231.

Dearing, J.A., Dann, R.J.L., Hay, K., Lees, J.A., 1996, Frequency-dependent susceptibility measurements of environmental materials: Geophysical Journal International, 124(1), 228-240.

Franke, C., Kissel, C., Robin, E., Bonté, P., Lagroix, F., 2009, Magnetic particle characterization in the Seine river system: Implications for the determination of natural versus anthropogenic input: Geochemistry, Geophysics, Geosystems, 10(8). doi:10.1029/2009GC002544.

Gaiero, D.M., Ross, G.R., Depetris, R.J.I., Kempe, S., 1997, Spatial and temporal variability of total non-residual heavy metals content in stream sediments from the Suquía river system, Córdoba, Argentina: Water, Air \& Soil Pollution, 93(1-4), 303-319.

Gordillo, C., 1972, Las rocas ortopiroxénicas de la Sierra de Córdoba: Boletín de la Asociación Geológica de Córdoba, 1(3-4), 111-115.

Gordillo, C., Lencinas, A., 1979, Sierras Pampeanas de Córdoba y San Luis: Geología Regional Argentina, 1, 577-650.

Harguinteguy, C.A., Cirelli, A.F., Pignata, M.L., 2014, Heavy metal accumulation in leaves of aquatic plant Stuckenia filiformis and its relationship with sediment and water in the Suquía river (Argentina): Microchemical Journal, 114, 111-118.

Herron, M.M., 1988, Geochemical classification of terrigenous sands and shales from core or log data: Journal of Sedimentary Research, 58(5), 820-829.

King, J., Banerjee, S.K., Marvin, J., Ozdemir, O., 1982, Relative grain size of magnetite in natural materials: some results from lake sediments: Earth and Planetary Science Letters, 59, 404-419.

Kottek, M., Grieser, J., Beck, C., Rudolf, B., Rubel, F., 2006, World map of the Köppen-Geiger climate classification updated: Meteorologische Zeitschrift, 15(3), 259-263.

Laveuf, C., Cornu, S., 2009, A review on the potentiality of Rare Earth Elements to trace pedogenetic processes: Geoderma, 154(1-2), 1-12.

Lecomte, K.L., Vignoni, P., Córdoba, F.E., Chaparro, M.A.E., Chaparro, M.A.E., Kopalová, K., Gargiulo, J.D., Lirio, J., Irurzun, M.A., Böhnel, H.N., 2016, Hydrological systems from the Antarctic Peninsula under climate change:
James Ross archipelago as study case: Environmental Earth Sciences, 75(7), 623, doi.org/10.1007/s12665-016-5406-y.

Liu, Q., Roberts, A.P., Larrasoaña, J.C., Banerjee, S.K., Guyodo, Y., Tauxe, L., Oldfield, F., 2012, Environmental magnetism: principles and applications: Reviews of Geophysics, 50(4), 1-50.

Locati, F., Colombo, F., Baldo, E., 2009, Pegmatitas con Allanita-(Ce) vinculadas al ortogneis piroxénico de la quebrada del río Suquía, Sierras Chicas de Córdoba: Revista de la Asociación Geológica Argentina, 64(4), 646-656.

Malpartida, A., 2003, La Cuenca del Rio Matanza-Riachuelo: Compuestos xenobióticos y otros polutantes en la cuenca: Revisión de Antecedentes de Recursos Naturales, Universidad Tecnológica Nacional, Argentina, 144 pp.

McLennan, S., 2001, Relationships between the trace element composition of sedimentary rocks and upper continental crust: Geochemistry, Geophysics, Geosystems, 2(4). doi.org/10.1029/2000GC000109.

Mejía-Echeverry, D., Chaparro, M.A.E., Duque-Trujillo, J.F., Chaparro, M.A.E., Castañeda-Miranda, A.G., 2018, Magnetic biomonitoring of air pollution in a tropical valley using a Tillandsia sp.: Atmosphere, 9(7), 283. doi:10.3390/atmos 9070000 .

Merlo, C., Abril, A., Amé, M.V, Argüello, G.A., Carreras, H.A., Chiappero, M.S., Hued, A.C., Wannaz, E., 2011, Integral assessment of pollution in the Suquía River (Córdoba, Argentina) as a contribution to lotic ecosystem restoration programs: Science of the Total Environment, 409, 5043-5045.

Meybeck, M., 2011, Global Occurrence of Major Elements in Rivers, in Holland H.D. and Turekian, K.K. (eds), Geochemistry of Earth Surface Systems: Amsterdam, Elsevier, Chapter 8, 277-292.

Monferrán, M.V., 2018, Metals and Metalloids, Water and Sediment of the Suquía River Basin: Spatial and Temporal Changes, in Wunderlin, D.A (ed.), The Suquía River Basin (Córdoba, Argentina): An Integrated Study on its Hydrology, Pollution, Effects on Native Biota and Models to Evaluate Changes in Water Quality: Switzerland, Springer, chapter 5, 113-144.

Muller, G., 1969, Index of geoaccumulation in sediments of the Rhine River: Geojournal, 2, 108-118.

Namiesnik, J., Szefer, P., 2009, Analytical measurements in aquatic environments: Boca Raton, Florida, CRC Press, 506 pp.

Otero, N., Vitorio, L., Soler, A., Canals, A., 2005, Fertilizer characterization major, trace and rare earth elements: Applied Geochemistry, 20, 1473-1478.

Papakostidis, G., Grimanis, A.P., Zafiropoulos, D., Griggs, G., Hopkins, T., 1975, Heavy metals in sediments from Athens sewage outfall area: Marine Pollution Bulletin, 6(9), 136-139.

Pasquini, A.I., Lecomte, K.L., Depetris, P.J., 2004, Geoquímica de ríos de montaña en las Sierras Pampeanas: II. El río Los Reartes, sierra de Comenchingones, provincia de Córdoba: Revista de la Asociación Geológica Argentina, 59(1), 129-140.

Pasquini, A.I., Formica, S.M., Sacchi, G.A., 2012, Hydrochemistry and nutrients dynamic in the Suquía River urban catchment's, Córdoba, Argentina: Environmental Earth Sciences, 65(2),453-467.

Pasquini, A., Campodonico, V., Rouzaut, S., Giampaoli, V., 2017, Geochemistry of a soil catena developed from loess deposits in a semiarid environment Sierra Chica de Córdoba, central Argentina: Geoderma, 295, 53-68.

Pesce, S., Wunderlin, D.A., 2000, Use of water quality indices to verify the impact of Córdoba City (Argentina) on Suquía River: Water Research, 34(11), 2915-2926.

Peters, C., Dekkers, M. J., 2003, Selected room temperature magnetic parameters as a function of mineralogy, concentration and grain size: Physics and Chemistry of the Earth, Parts A/B/C, 28(16-19), 659-667.

Pettijohn, F.J., Potter, P.E., Siever, R., 1972, Sand and Sandstone: Springer-Verlag, Berlin-Heidelberg, $618 \mathrm{pp}$.

Puig, A., Olguín, H., 2006, Cursos fluviales bonaerenses: estrés químico y ecohidrología, in Capaldo, G. (ed.), Sinergias Ambientales entre las Aguas Continentales y las Marinas: Buenos Aires, Mare Magnum, 55-67.

Quijano, L., Chaparro, M.A.E., Marié, D.C., Gaspar, L., Navas, A., 2014, Relevant magnetic and soil parameters as potential indicators of soil conservation status of Mediterranean agroecosystems: Geophysical Journal International, 198(3),1805-1817.

Rapela, C.W., Pankhurst, R.J., Casquet, C., Baldo, E.G., Saavedra, J., Galindo, C., Fanning, C.M., 1998, The Pampean Orogeny of the southern protoAndes: Cambrian continental collision in the Sierras de Cordoba: London, Geological Society Special Publications, 142(1), 181-217.

Salbu, B., Krekling, T., Oughton, D.H., 1998, Characterization of radioactive 
particles in the environment: The Analyst, 123, 843-850.

Santa Cruz, J., 1972, Geología al Este de la Sierra Chica (Córdoba), Valle del Río Primero: Boletín de la Asociación Geológica de Córdoba, I (3-4), 102-109.

Snowball, I., Zillén, L., Gaillard, J., 2002, Rapid early Holocene environmental changes in northern Sweden based on studies of two varved lake-sediment sequences: The Holocene, 12(1),7-16.

Stupar, Y.V., Schäfer, J., García, M.G., Schmidt, S., Piovano, E., Blanc, G., Huneau, F., Coustumer, P. Le, 2013, Historical mercury trends recorded in sediments from the Laguna del Plata, Córdoba, Argentina: Chemie der Erde - Geochemistry, 74(3), 353-363.

Sutherland, R., 2000, Bed sediment-associated trace metals in an urban stream, Oahu, Hawaii: Environmental Geology, 39(6), 611-627.

Szefer, P., Glasby, G.P., Kusak, A., Szefer, K., Jankowska, H., Wolowicz, M., Ali, A.A., 1998, Evaluation of the anthropogenic influx of metallic pollutants into Puck Bay, southern Baltic: Applied Geochemistry, 13(3), 293-304.

Ure, A., Quevauviller, P., Muntau, H., Griepink, B., 1993, Speciation of heavy metals in soils and sediments. An account of the improvement and harmonization of extraction techniques undertaken under the auspices of the BCR of the Commission of the European Communities: International Journal of Environmental Analytical Chemistry, 51(1-4), 135-151.

Valentinuzzi, M.C., Sánchez, H.J., Abraham, J., 2006, Total reflection X-ray fluorescence analysis of river waters in its stream across the city of Cordoba in Argentina: Spectrochimica Acta, Part B, Atomic Spectroscopy, 61, 1175-1179.
Vargas, T. de, 2010, Transferência de elementos maiores, traços e elementos das terras raras no sistema fertilizante - solo - água subterrânea em sedimentos Quaternários da região de Águas Claras, município de Viamão, RS: Porto Alegre, Universidade Federal do Rio Grande do Sul, Instituto de Geociências, tesis de maestría, 103 pp.

Wunderlin, D., 2018, The Suquía River Basin (Córdoba, Argentina): An Integrated Study on its Hydrology, Pollution, Effects on Native Biota and Models to Evaluate Changes in Water Quality: Switzerland, Springer, $207 \mathrm{pp}$.

Wunderlin, A.D., Díaz, M., Amé, M. V., A, Pesce, S.F., Hued, A.C., 2001, Pattern recognition techniques for the evaluation of spatial and temporal variations in water quality. A case study: Suquía River basin (Córdoba - Argentina): Water Research, 35(12), 2881-2894.

Yang, T., Liu, Q., Chan, L., Liu, Z., 2007, Magnetic signature of heavy metals pollution of sediments: case study from the East Lake in Wuhan, China: Environmental Geology, 52, 1639-1650.

Manuscrito recibido: agosto 23, 2018

Manuscrito corregido recibido: febrero 19, 2019

Manuscrito aceptado: febrero 20, 2019 